Relations industrielles

Industrial Relations

\title{
L'Université et les relations industrielles
}

\section{Georges-Henri Lévesque}

Volume 1, numéro 7, mars 1946

URI : https://id.erudit.org/iderudit/1023942ar

DOI : https://doi.org/10.7202/1023942ar

Aller au sommaire du numéro

Éditeur(s)

Département des relations industrielles de l'Université Laval

ISSN

0034-379X (imprimé)

1703-8138 (numérique)

Découvrir la revue

Citer cet article

Lévesque, G.-H. (1946). L'Université et les relations industrielles. Relations

industrielles / Industrial Relations, 1(7), 6-7. https://doi.org/10.7202/1023942ar

Tous droits réservés @ C Département des relations industrielles de l’Université Laval, 1946
Ce document est protégé par la loi sur le droit d'auteur. L'utilisation des services d'Érudit (y compris la reproduction) est assujettie à sa politique d'utilisation que vous pouvez consulter en ligne.

https://apropos.erudit.org/fr/usagers/politique-dutilisation/ 


\section{L'UNIVERSITÉ ET LES RELATIONS INDUSTRIELLES}

Sera-t-il dit que devant un appel aussi pressant l'Université va rester insensible et se reconnaître impuissante? Non, car s'il est vrai que les Relations industrielles ont besoin de l'Université, il ne l'est pas moins que l'Université, de son côté, peut répondre à ce besoin.

Celle que l'on a appelée " école de haut savoir et source de directives sociales ", on la nomme aussi le cerveau de la nation. Pour répondre adéquatement aux besoins de celle-ci, ce cerveau possedde en aussi grand nombre qu'il en faut des Facultés. Par exemple, la Faculté de médecine pour veiller sur la santé physique du corps social; la Faculté de droit pour établir les cadres juridiques de la vie nationale et assurer le règne de la justice entre les citoyens ; la Faculté de philosophie qui recherche et diffuse les lumières de la sagesse ; la Faculté de théologie pour subvenir aux besoins spirituels les plus élevés; la Faculté des sciences qui cherche -à découvrir les lois de la nature pour mettre celle-ci plus entièrement et plus efficacement au service de l'homme.

Si donc l'Université a raison de s'occuper de la vie religieuse, intellectuelle, juridique, physique et matérielle de la nation, n'est-elle pas aussi justifiée de veiller sur sa vie sociale, toute faite de relations : d'abord entre les individus, puis entre ces derniers et les sociétés et enfin entre les sociétés elles-mêmes ? Voilà bien pourquoi elle a sa Faculté des sciences sociales, qui forme elle aussi des "professionnels", dont la tâche propre est de chercher à créer ou à maintenir dans la société de bonnes relations. Et puisque parmi les relations sociales, les relations industrielles présentent des difficultés spéciales, il était normal que cette Faculté mît sur pied un Département des relations industrielles pour travailler à résoudre le mieux possible ces difficultés.

Mais alors, plus précisément, de quelle façon l'Université peut-elle contribuer à de meilleures relations industrielles?

D'abord, par ses travaux de recherche. Lorsqu'on se propose, en effet, d'améliorer un objet quelconque, la première chose à faire est de le bien connaître, de l'étudier positivement, d'en saisir de façon bien réaliste la nature et les diverses propriétés.

Or, est-il institution mieux placée que l'Université pour étudier efficacement et dans toute leur ampleur, les réalités sociales? Elle a pour cela toutes les connaissances voulues ayant à sa portée les secours de la philosophie, de la sociologie, de la doctrine sociale de l'Eglise, comme aussi de toutes les sciences connexes

(1) Voici le texte de la seconde partie de l'allocution prononcée par le T. R. P. Georges-Henri Ltevesque, o.P., doyen de la Faculté des Sciences sociales de Laval, à l'ouverture du Congrès des relations industrielles au Château Frontenac. Le texte intégral da cette allocution paraîtra dans le compte-rendu du Congrès qui sera imprimé sous peu, qu'elle peut utiliser à son gré. Elle a de plus toutes les techniques scientifiques qui conviennent. Ses facilités d'observation sont immenses, elle peut faire de vastes enquêtes sociales, s'appuyer sur des statistiques scientifiquement compilées et interprétées. De plus, et cela est bien précieux, c'est elle qui offre les meilleures garanties d'impartialité, puisqu'elle jouit de toute l'indépendance et de l'éloignement nécessaires à une juste appréciation des hommes et des choses. Située sur un sommet, elle a une meilleure vue sur les ensembles et ses perspectives sont d'autant plus réalistes qu'elle voit les problèmes particuliers en fonction de l'ordre général. Mais si haut qu'elle plonge son règard, elle n'est cependant pas pour autant isolée du réel concret, comme on peut être quelquefois enclin à le lui reprocher. Au contraire, elle s'efforce de maintenir un contact très étroit et de garder des relations constantes avec les entreprises et la vie industrielle elle-même; elle s'applique aussi à se tenir très près des groupes ouvriers ou patronaux, à l'aide desquels, d'ailleurs, elle aime à conduire ses plus fructueuses expériènces sociales. Le seul fait du présent congrès n'en est-il pas un magnifique exemple? Et s'il en fallait un autre, je le trouverais facilement dans ceci que nos professeurs se trouvent à peu près tous engagés de quelque façon dans la vie sociale industrielle par les travaux qu'ils accomplissent, soit pour les patrons, soit pour les unions ouvrières, soit pour l'Etat. C'est donc dire que par ses travaux de recherches, l'Université peut rendre de très grands services aux relations industrielles. C'est là ce que j'appellerais volontiers le moment sociologique de son action.

Il en est un autre, cependant, plus proprement social, celui-là, et certes non moins important : celui de la formation de spécialistes en relations industrielles. L'Université prépare tout d'abord des agents de relations industrielles, à qui elle donne toute la formation doctrinale, sociologique, technique, psychologique et morale voulue pour qu'ils deviennent de véritables instruments de coopération, d'harmonie et de paix sociales et dont l'action s'inspirera non pas des intérêts de tel ou tel groupe (patrons ou ouvriers), mais du bien commun de toute la société. Car, ne l'oublions jamais, il y a, dans tout conflit industriel, un tiers intéressé autant qu'invisible mais plus important peut-être que les deux autres et dont la présence réelle doit se faire sentir dans les conseils industriels: c'est la société, le peuple, la masse anonyme des consommateurs dont nous sommes tous et sur le dos de qui se règlent malheureusement trop souvent maintes querelles sociales. Il ne faut pas perdre de vue que les professions et les métiers existent pour faire vivre leur homme, sans doute, mais aussi et beaucoup pour faire vivre les hommes en société. C'est là l'intention formelle et bien explicite de la grande loi naturelle de la division du travail. Et c'est précisément pour cela que la question des rapports entre le capital et le travail s'appelle partout, et avec un sens 
très profond, la question sociale : la société y est aussi directement intéressée que le capital et le travail.

Mais l'Université ne se borne pas à former des agents de collaboration, elle forme aussi des chefs pour les organisations ouvrières. Par son enseignement proprement universitaire d'abord, et ensuite par le truchement de son Service extérieur d'éducation sociale, elle s'efforce de préparer, pour les syndicats ouvriers, des hommes véritablement aptes à défendre, et le mieux possible, les intérêts bien compris et les droits inaliénables de la personne ouvrière. De vrais chefs qui seront non seulement les porte-parole éclairés des justes revendications de la classe ouvrière, mais aussi, les éducateurs sociaux qui donneront aux travailleurs le souci de leur grande dignité, tout en leur apprenant à maintenir leurs exigences dans les normes de la justice, à juger de leurs problèmes en fonction du bien commun et à maintenir sous le contrôle de la prudence, sans pour autant les paralyser, leur dynamisme spontané et leur naturelle tendance à la revendication.

L'Université prépare enfin, pour les patrons, les experts dont ils ont besoin. Ces hommes apprendront à l'Université que si les patrons ont des devoirs ils ont aussi des droits, que s'ils ont des droits ils ont aussi des devoirs; que la propriété est un droit naturel, mais qu'elle a aussi ses charges sociales; que les ouvriers ne sont pas des machines et que leur travail n'est pas une vulgaire marchandise ; que le travailleur est une personne humaine, et que, de ce fait, les conditions de son travail ne doivent pas être celles des esclaves ; mais des hommes libres, égaux en dignité de nature, créés à l'image de Dieu et rachetés par son sang.

Voilà, mesdames et messieurs, trop brièvement rappelé, le rôle de l'Université dans le domaine des relations industrielles. Avons-nous assez souligné, cependant, le caractère essentiellement spirituel et moral de ces relations? C'est là un fait qui rend encore plus nobles et plus pressantes les tâches que nous venons d'assigner à l'Université ; et c'est sur cette dernière considération que je veux achever mon discours. Les temps mémorables que nous vivons sont témoins d'un retour offensif du matérialisme révolutionnaire. On dirait que les hommes ont perdu de vue les fins de la vie humaine pour se rabattre sur la seule contemplation des moyens. L'intelligence humaine dont les lumières ont pu percer les secrets les plus cachés du monde physique semble bien pauvrement outillée pour assurer à l'humanité un progrès social et moral comparable à ses progrès matériels. Serait-ce vraiment que la matière est la seule réalité qui existe et au-delà de laquelle il n'y a rien que superstitions religieuses ou autres? Bien des faux docteurs le prétendent aujourd'hui, et avec d'autant plus de chance d'être crus que les forces spirituelles semblent accuser un scandaleux recul. $\mathrm{Si}$ l'homme contemporain ne retrouve pas bientôt son âme, il est à craindre qu'il ne périsse sous les décombres d'une civilisation que les plus belles puissances intellectuelles et morales de ses ancêtres ont mis des siècles à construire, et que son incompréhensible égarement menace de laisser détruire en un instant.

Si le siècle qui a vu, comme pas un autre, le fléau des crises économiques et des grands conflits industriels, à qui fut donné le spectacle des deux plus horribles guer- res qui aient jamais ensanglanté la terre des hommes, qui voit présentement se dresser au lendemain même de l'hécatombe, dominant celui de la famine, le spectre plus épouvantable encore d'une troisième guerre mondiale, si ce siècle, dis-je, ne revient à la foi en l'esprit, il faudra désespérer de la destinée humaine. Ah ! si les hommes mettaient autant d'amour à développer leurs (nergies spirituelles pour le plus grand bien de toute la société qu'ils mettent d'intelligence à harnacher celles de la matière pour la destruction de leurs frères, comme le monde serait plus heureux. Si au lieu des chəmps de bataille, où l'on se tue, il n'y avait que des champs de travail où, loin de se combattre, l'on peinerait ensemble les uns pour les autres ; si les humains savaient retrouver dans leur détresse même les raisons de leur fraternité perdue ; s'ils voulaient croire un instant aux promesses de la justice, et s'ils pouvaient surtout, oui s'ils pouvaient jamais communier aux douceurs bienfaisantes de la charité chrétienne, comme la vie serait plus belle partout, dans les chantiers de l'industrie comme sur la scène du monde! Il est bien malheureux que nous soyons si loin de tout cela, mais rien n'est perdu tant que des hommes restent capables de se rencontrer sérieusement, comme vous le faites en ce moment, tant que vacille encore au fond troublé des consciences humaines la moindre lueur de foi en des jours meilleurs. Ce n'est certes pas nous, chrétiens, qui croyons à l'existence de'la Providence et à la bonté d'un Père qui n'abandonne jamais ses enfants, ce n'est pas nous qui manquerons d'espérance.

Il faut croire au contraire que dans l'abîme profond de son malheur, à la terrible lumière des catastrophes qui ont précipité sa chute, l'homme saura reprendre la main blessée de son frère et sans regarder les ruines, oubliant dans un grand pardon toutes les haines du passé, marcher avec lui sur le sentier si longtemps désert mais désormais retrouvé de la paix, cette tranquillité de l'ordre.

\section{L'ATELIER PRÉFÉRENTIEL}

(Suite de la page \&)

puis les syndiqués, en tenant compte de leur expérience et de leur droit d'ancienneté."

“ L'employeur convient de n'embaucher aucun employé tant qu'un ou plusieurs des membres du syndicat, déjà mis à pied par l'employeur pour cause de diminution de travail, n'ont pas repris celui-ci. Si ce ou ces membres du syndicat, après avoir reçu un avis, négligent de reprendre leur travail dans les 48 heures, l'employeur pourra embaucher les candidats qu'il désirera."

\section{Promotions:}

"Lorsqu'une position deviendra vacante, une préférence sera accordée aux ouvriers membres en règle avec le Syndicat, mais en tenant compte de leur expérience, de leur compétence, ainsi que de leur droit d'ancienneté."

Gerard Dion. 